\title{
A Procedure for Predicting the Heat Release Rate of Furniture Composites from Measurements on their Components
}

\author{
WILLIAM J. PARKER ${ }^{1}$ \\ Fire Technology Consultant \\ 13135 Dairymaid Drive T-2 \\ Germantown, Maryland 20874 USA
}

\begin{abstract}
A computer model was developed for predicting the heat release rate versus time curves for furniture composites in the Cone calorimeter, based on the measured heat release rate curves of the individual components using modified test procedures. The composite model can be used for both melting and charring fabrics but is restricted to melting foams. The validation for the padding was limited to three different types of polyurethane foams. Making measurements on the components rather than directly on the composites substantially reduces the amount of testing required. Furthermore, the testing can be done by the material suppliers rather than by the furniture manufacturers.
\end{abstract}

KEYWORDS: composite model; computer model; Cone calorimeter; fabric; furniture; furniture composite; heat release rate; polyurethane foam.

\section{NOTATION}

$f_{1} \quad$ average sensitivity of the foam before melting is complete (-)

$\mathrm{f}_{2} \quad$ sensitivity of the foam at the time of the peak heat release rate

$\mathrm{g}_{1} \quad$ shape function of the foam before melting is complete (assumed to be 1.0)

$\mathrm{g}_{2} \quad$ shape function of the foam after melting is complete

I integrated heat release rate of foam up to time $t(\mathrm{~kJ})$

$\mathrm{I}_{\mathrm{A}} \quad$ value of I when the heat release rate increases to half of its maximum value $(\mathrm{kJ})$

${ }^{1}$ This work was conducted while the author was at SP, Swedish National Testing and Research Institute in Borås, Sweden. 
$\mathrm{I}_{\mathrm{B}} \quad$ value of I when the heat release rate falls to half of its maximum value $(\mathrm{kJ})$

$\mathrm{I}_{\mathrm{pk}} \quad$ value of I when the heat release rate reaches its maximum value $(\mathrm{kJ})$

$\mathrm{I}_{\mathrm{T}} \quad$ value of $\mathrm{I}$ at the time that the melting is complete $(\mathrm{kJ})$

$\mathrm{k}$ numerical constant $\left(\mathrm{m}^{4} / \mathrm{kJ}^{2}\right)$

$\dot{q}_{\text {comp }}^{\prime \prime} \quad$ heat release rate of the composite $\left(\mathrm{kW} / \mathrm{m}^{2}\right)$

$\dot{q}_{f a b}^{\prime \prime} \quad$ heat release rate of the fabric $\left(\mathrm{kW} / \mathrm{m}^{2}\right)$

$\dot{q}_{\text {fom }}^{\prime \prime} \quad$ heat release rate of the foam $\left(\mathrm{kW} / \mathrm{m}^{2}\right)$

$\dot{q}_{\text {fompk }}^{\prime \prime}$ peak heat release rate of the foam $\left(\mathrm{kW} / \mathrm{m}^{2}\right)$

$\dot{q}_{\text {fom,shld }}^{\prime \prime}$ heat release rate of the foam before melting is complete $\left(\mathrm{kW} / \mathrm{m}^{2}\right)$

$\dot{q}_{\text {std.comp }}^{\prime \prime}$ heat release rate of the standard composite $\left(\mathrm{kW} / \mathrm{m}^{2}\right)$

$\dot{q}_{\text {std fom }}^{\prime \prime}$ heat release rate of the standard foam $\left(\mathrm{kW} / \mathrm{m}^{2}\right)$

$\mathrm{S}_{1} \quad$ sensitivity of the foam before melting is complete (-)

$\mathrm{S}_{2} \quad$ sensitivity of the foam after melting is complete (-)

$\mathrm{S}_{\mathrm{fom}} \quad$ sensitivity of the foam (-)

$\mathrm{S}_{\text {std. fom }}$ sensitivity of the standard foam (-)

$\mathrm{t} \quad$ time (s)

$\mathrm{t}_{\mathrm{T}} \quad$ time at which melting is complete (s)

$\phi_{\text {ext }} \quad$ external radiant flux $\left(\mathrm{kW} / \mathrm{m}^{2}\right)$

$\phi_{f r} \quad$ flame radiation incident on the foam $\left(\mathrm{kW} / \mathrm{m}^{2}\right)$

$\phi_{\text {loss }} \quad$ heat losses from the foam $\left(\mathrm{kW} / \mathrm{m}^{2}\right)$

$\phi_{\text {net }} \quad$ net heat flux into the foam $\left(\mathrm{kW} / \mathrm{m}^{2}\right)$

\section{INTRODUCTION}

A procedure was developed for predicting the heat release rate of furniture composites in the Cone calorimeter [1], based on the measured heat release rates of the individual furniture components (i.e. foams, fabrics and interliners if any). It predicts the complete heat release rate curve that would be needed for input to the furniture fire models. It requires special testing protocols for the components. This was part of a much larger research project on the Combustion Behavior of Upholstered Furniture (CBUF) [2] carried out for the Commission of the European Communities.

There is a standard method [3] for measuring the heat release rate of furniture composites in the Cone calorimeter. This standard provides detailed instructions on specimen preparation based on round robin tests [4] in conjunction with the CBUF project. The $100 \mathrm{~mm}$ by $100 \mathrm{~mm}$ by 50 $\mathrm{mm}$ thick block of padding is enclosed on the sides and the top surface by the fabric and the interliner, if any, that would be present in the full scale furniture. This assembly is then wrapped in aluminum foil except for the top surface which is exposed from above to the thermal radiation from the cone heater. This wrapping provides an open box or cup which retains all of the melting materials throughout the burning process.

The data on the components were obtained at the Dansk Brandteknisk Institut (DBI) in Denmark and at the FMC Corporation Ltd. (FMC) in England. The comparison data on the composites were obtained at the Forschungs-und Materialprufungsanstalt Baden-Wurttemberg (FMPA) in Germany and at the Laboratories National d'Essais (LNE) in France. 


\section{BURNING BEHAVIOR}

An exploratory series of tests, some of which were instrumented with thermocouples and heat flux meters, were conducted to gain a better understanding of the burning behavior of the individual components and their interactions. These tests formed the basis for the assumptions used in the model and for the development of the component testing procedures. This work was done at the Swedish National Testing and Research Institute (SP) and at DBI.

\section{Charring fabrics}

When cotton burns it releases volatiles which have a relatively low heat of combustion since much of the carbon is held back in the formation of the char. The fabric shrinks as it chars but it still retains the original weave pattern and the shape of the fabric shell. It undergoes flaming combustion as long as a sufficient flow of volatiles is maintained. Whenever flaming stops in some local area, glowing combustion takes over there due to char oxidation. Thus the heat release rate of a cotton fabric burning by itself is due to flaming at first, then a mixture of flaming and glowing, and finally all glowing. However, if it is over a polyurethane foam, the flow of volatiles passing through the fabric throughout the test will maintain a flame above the surface that will consume the oxygen and prevent char oxidation.

In order to test a fabric by itself and provide information on how it would burn if it were part of a composite, a method of simulating the volatiles from the foam had to be devised. The method chosen was to test the fabric with a flow of methane passing through it. When this is done there is always a flame above the surface of the specimen. This flame prevents oxygen from diffusing in to the surface and thus avoids the glowing combustion. Under these conditions, the fabric should burn like it would in a composite.

Tests were run on fire retardant cotton covers with and without methane flowing through them. During its fully involved flaming period the heat release rate of the fabric cover was not significantly affected by the presence of the gas. After its flaming contribution was over, the heat release rate due to the cover dropped to zero if methane was flowing through it. After the gas was turned off, the cover released almost as much heat due to glowing combustion as it had done previously due to flaming. With the higher emissivity of the volatiles released by the melting foam, one might expect that it could have more impact than the methane on the flaming contribution of the fabric. However, as will be seen below, the heat release rate of the foam is quite low while it is still in its melting phase. This phase is generally longer than the flaming period of the fabric. Furthermore, there has generally been good agreement between the predicted and measured first peaks in the heat release rate of the composites.

The three main aspects of the combustion behavior of a char-forming fabric are (1) to produce a large part of the initial peak heat release rate, (2) to act as a thermal radiation shield to severely limit the heat flux to the melting foam and (3) to prevent the penetration of the flames into the region near the surface of the foam, thus cutting out direct flame heat transfer.

\section{Melting fabrics}

The melting fabric quickly falls onto the top of the foam and forms a pool there. The heat flux 


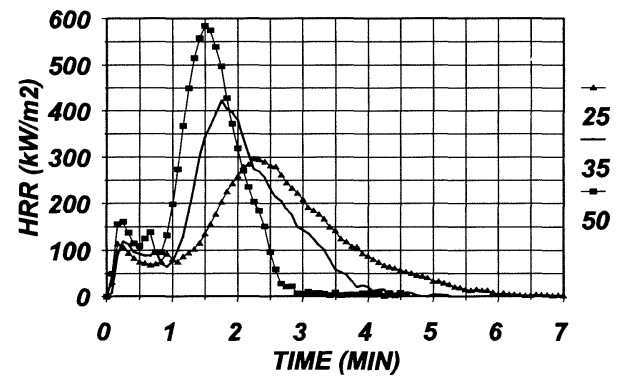

FIGURE 1. Heat release rates of bare CMHR foam at 25,35 and $50 \mathrm{~kW} / \mathrm{m}^{2}$

actually reaching the foam is reduced by the energy consumed in the vaporization of the fabric pool during the early part of the test. After the melted fabric is gone, nearly all of the heat flux reaching the opening at the top of the aluminum foil cup is transmitted, either directly or by reflection from the foil walls, to the foam. At low mass loss rates the flames approach the pool at the bottom of the cup and transfer additional heat by convection.

\section{Foams}

The foam burns in two stages which are clearly seen in the heat release rate curves in Figure 1 for a bare CMHR (combustion modified high resilience polyurethane) foam at external fluxes of 25,35 and $50 \mathrm{~kW} \mathrm{~m}^{-2}$. Decomposition, melting, and volatilisation take place during the collapse of the $50 \mathrm{~mm}$ thick block to a shallow pool of combustible liquid at the bottom of the aluminium foil cup during the first stage which lasts of the order of $30 \sim 60 \mathrm{~s}$ for a foam burning by itself. It is extended when the foam is protected by a charring fabric. This phase is characterised by a low heat release rate since much of the incoming heat flux is consumed in the degradation and melting processes. The beginning of the second stage is marked by a rapid increase in heat release rate from the pool fire at the bottom of the aluminium foil cup. Since the melting is complete, nearly all of the incoming heat flux is going into the evaporation of the liquid. Most of the heat is released during this second stage.

\section{FORMULATION}

The heat release rate of the furniture composite is equal to the sum of the heat release rate contributions of the individual components.

$\dot{q}_{\text {comp }}^{\prime \prime}=\dot{q}_{f o m}^{\prime \prime}+\dot{q}_{f a b}^{\prime \prime}$ 
The individual heat release rate contributions of the fabric and the foam used in Eq. 1 must be the same as if they were burned together in the composite. All of the heat release rates referred to in this paper are functions of time except for the peak heat release rate which depends only on the net flux. It is understood to be the peak heat release that would have been obtained if that flux were maintained over the entire exposure period.

The test for the fabric is conducted with methane flowing through it to simulate the effect of the volatiles from the foam. The heat release rate of the methane is subtracted from the total measured heat release rate to yield the heat release rate of the fabric as if it were burning over a foam.

The heat release rate contributed by the foam is equal to the product of its sensitivity and the net heat flux absorbed by it. Thus,

$$
\dot{q}_{\text {fom }}^{\prime \prime}=S_{\text {fom }} \dot{q}_{\text {net }}^{\prime \prime}
$$

The sensitivity is determined by testing the bare foam. The heat release rate curves for CMHR foam at three different fluxes are shown in Figure 1. The sensitivity is found by dividing the heat release rate of the foam by the net heat flux absorbed by it. In determining the sensitivity, it is necessary to consider the low, relatively flat shoulder region at the beginning of the test and the main region separately because of the difference in their burning behavior. Thus, $\mathrm{S}_{\mathrm{fom}}$ is defined as $S_{1}$ in the shoulder region and $S_{2}$ in the main region. $S_{2}$ is given by

$$
S_{2}=\frac{\dot{q}_{f o m}^{\prime \prime}}{\phi_{n e t}}
$$

Note that $S_{2}$ is equal to the heat of combustion divided by the effective heat of gasification of the foam and varies with time. While the true heat of gasification is a constant, the effective heat of gasification includes the effect of interior heat conduction and heat losses through the rear surface.

Eq. 3 can be expanded in the following way:

$$
S_{2}=\frac{\dot{q}_{f o m, p k}^{\prime \prime}}{\phi_{n e t}} \frac{\dot{q}_{f o m}^{\prime \prime}}{\dot{q}_{f o m, p k}^{\prime \prime}}=f_{2} g_{2}
$$

where

$$
f_{2}=\frac{\dot{q}_{f o m, p k}^{\prime \prime}}{\dot{q}_{n e t}}
$$

is the sensitivity of the foam at its peak heat release rate and 
$g_{2}=\frac{\dot{q}_{f o m}^{\prime \prime}}{\dot{q}_{f o m, p k}^{\prime \prime}}$

is a function, running from 0 to 1.0 , which describes the shape of the sensitivity curve.

First the function, $\mathrm{f}_{2}$, will be determined. The net heat flux absorbed by the bare foam is equal to the external flux plus the flame radiation minus the heat losses.

$\phi_{n e t}=\phi_{e x t}+\phi_{f r}^{\prime \prime}-\phi_{l o s s}^{\prime \prime}$

The peak sensitivity is then given by

$$
f_{2}=\frac{\dot{q}_{f o m, p k}^{\prime \prime}}{\Phi_{e x t}+\left[\phi_{f r}-\phi_{l o s s}\right]}
$$

The quantity in the brackets is not known in advance. However, it can be determined by plotting the peak heat release rate as a function of the external flux as done for the upper line in Figure 2 for polyether foam. It is equal to the magnitude of the intercept on the horizontal axis but of opposite sign. This determination would require testing the foam at three different fluxes. Therefore, another approach was adopted.

In most cases the flame radiation is significantly larger than the heat losses. The quantity in the brackets could be brought to a sufficiently small value to neglect, if the flame radiation could be controlled at a very low level. This was accomplished by introducing methane into the region just above the specimen. The methane flame has a relatively low emissivity. It displaces the highly radiative flame produced by the volatiles from the decomposing and melting foam. These volatiles burn much higher up in the flame when the methane is present and only a small fraction of their radiated heat reaches the specimen. The effectiveness of this technique is demonstrated by the lower line in Figure 2 where measurements using methane produced intercepts much closer to the origin for tests conducted at DBI and FMC. This was also observed for the HR (high resilience polyurethane) and CMHR foams. Under these conditions it is possible to determine $f_{2}$ with only a single test at $35 \mathrm{~kW} / \mathrm{m}^{2}$. Thus,

$$
f_{2}=\frac{\dot{q}_{f o m, p k}^{\prime \prime}}{35}
$$

A methane flow capable of producing a measured heat release rate of $370 \mathrm{~kW} \mathrm{~m}{ }^{-2}$ with no specimen in place was found to be sufficient. The methane is also effective in keeping the oxygen from diffusing to the bottom of the aluminum foil cup and producing flames close to the foam when its mass loss rate is small. When the flames approach the pool, there is a large 


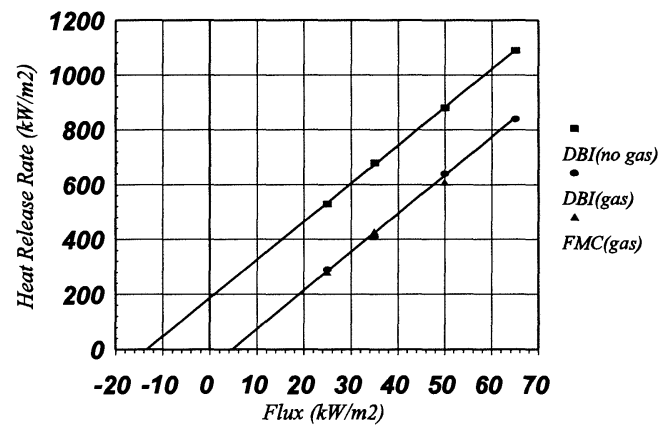

FIGURE 2. Peak heat release rate of polyether foam as a function of flux.

varying and unknown convective component of the heat transfer to the surface of the pool that needs to be avoided.

The shape function, $\mathrm{g}_{2}$, in the main, or pool burning, region is given by Eq. 6 . The heat release rate of the CMHR foam was plotted as a function of time for three fluxes in Figure 1. Each of the curves is divided by its peak heat release rate and plotted as a function of I in Figure 3 where $\mathrm{I}$ is the integrated heat release up to the time, $\mathrm{t}$.

It is seen that if $g_{2}$ is expressed as a function of I instead of $t$, it is essentially independent of the external flux in the main part of the curve, a requirement that must be satisfied if the foam test is to be run only at one flux since the flux seen by the foam, when it is a part of the composite, varies with time throughout the burning period. The integral, $I$, is given by

$I=\int_{0}^{t} \dot{q}_{f o m}^{\prime \prime} d t$

The main curve can be represented fairly well by

$g_{2}=1-k\left(I-I_{p k}\right)^{2}$

where $\mathrm{k}$ is a curve-fitting coefficient and $\mathrm{I}_{\text {peak }}$ is the value of $I$ for which $\mathrm{g}_{2}$ has its maximum value. Since the top of the curve is relatively flat, $\mathrm{I}_{\text {peak }}$ is not easy to determine. It is better to define it as

$$
I_{p k}=\frac{\left(I_{A}+I_{B}\right)}{2}
$$




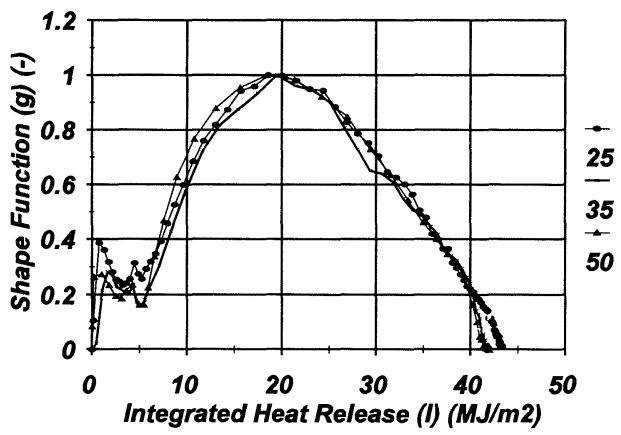

FIGURE 3. Shape function for sensitivity of bare CMHR foam at three fluxes.

where $I_{A}$ is the value of $I$ for which the curve rises to half of its maximum value and $I_{B}$ is the value of I for which the curve descends to half of its maximum value. By substituting Eq. 12 into Eq. 11 and setting $g_{2}\left(I_{A}\right)=0.5, k$ can be determined and substituted into Eq. 11 to yield

$$
g_{2}(I)=1-\frac{\left(2 I-I_{B}-I_{A}\right)^{2}}{2\left(I_{B}-I_{A}\right)^{2}}
$$

$I_{A}$ and $I_{B}$ are the two parameters that need to be measured for each foam in order to determine $\mathrm{g}_{2}(\mathrm{I})$.

The heat release rate of the shoulder takes on a variety of shapes but without any obvious functional dependence. It is best to approximate it by a constant value so that the shape factor, $\mathrm{g}_{1}$, is equal to unity. Since the foam test is run at $35 \mathrm{~kW} \mathrm{~m}^{-2}$, the average sensitivity in the shoulder region is then given by

$f_{1}=\frac{\dot{q}_{\text {fom }, \text { shld }}^{\prime \prime}}{35}=\frac{I_{T}}{35 t_{T}}$

where $I_{T}$ is the integrated heat release in the shoulder region and $t_{T}$ is the time of transition between the two regions. The time is measured from the instant of ignition. The time, $t_{\mathrm{T}}$, is defined experimentally by observing the time at which the heat release rate starts to rise monotonically toward the peak as seen in Figure 1.

The sensitivity of the foam is then given by

$S_{\text {fom }}=\frac{I_{T}}{35 t_{T}}$ for $I \prec I_{T}$
$S_{\text {fom }}=f_{2} g_{2}$ for $I \succeq I_{T}$ 
The sensitivity, $\mathrm{S}_{\text {fom }}$, must be multiplied by the net flux to get the heat release rate of the foam according to Eq. 2. During the burning of a composite the net heat flux defined in Eq. 7 for bare foams is modified by the presence of the fabric. Thus the net flux depends on the fabric and is not possible to calculate routinely. It must be measured.

In order to determine the net heat flux associated with a particular fabric, that fabric cover is tested over a standard foam of known sensitivity. The composite that is formed in this way is referred to below as the standard composite. The net heat flux is given by

$$
\phi_{\text {net }}=\frac{\dot{q}_{\text {std.foam }}^{\prime \prime}}{S_{\text {std.foam }}^{\prime \prime}}
$$

The numerator is the heat release rate contribution of the standard foam when it is covered with the fabric that would be used in the composite to be predicted. However, the measured heat release rate is that of the standard composite. The heat release rate of the fabric burning alone must be subtracted from it to get the heat release rate contribution of the standard foam.

$\dot{q}_{\text {std. fom }}^{\prime \prime}=\dot{q}_{\text {std. comp }}^{\prime \prime}-\dot{q}_{f a b}^{\prime \prime}$

Hence,

$\phi_{\text {net }}=\frac{\left(\dot{q}_{\text {std. comp }}^{\prime \prime}-\dot{q}_{f a b}^{\prime \prime}\right)}{S_{\text {std.foam }}}$

Here the standard foam is essentially being used as a simulated heat flux transducer.

Eq. 18 can be substituted into Eq. 2 to obtain the heat release rate contribution of the foam.

$\dot{q}_{f o m}^{\prime \prime}=\frac{S_{f o m}}{S_{\text {std. fom }}}\left(\dot{q}_{\text {std. comp }}^{\prime \prime}-\dot{q}_{f a b}^{\prime \prime}\right)$

where the sensitivities, S, are defined by Eqs. 15, 9, 10 and 13.

The heat release rate of the fabric is then added to get the predicted heat release rate of the composite as formulated in Eq. 1.

$\dot{q}_{f o m}^{\prime \prime}=\frac{S_{f o m}}{S_{s t d . f o m}}\left(\dot{q}_{s t d . c o m p}^{\prime \prime}-\dot{q}_{f a b}^{\prime \prime}\right)+\dot{q}_{f a b}^{\prime \prime}$

All of the quantities in Eq. 20 are functions of time. 


\section{CALCULATION}

The basic equation for the calculation of the predicted heat release rate contribution of the foam is Eq.19. The sensitivities $S_{\text {fom }}$ and $S_{\text {std. fom }}$ are functions of I which have been determined from the bare foam tests using Eq. 15. The foam parameters, $\mathrm{t}_{\mathrm{T}}, \mathrm{I}_{\mathrm{T}}, \dot{q}_{p k}^{\prime \prime}, \mathrm{I}_{\mathrm{A}}$ and $\mathrm{I}_{\mathrm{B}}$ required by equation 15 can be determined by a subroutine that operates on the complete heat release rate curves for the bare foams. The value of I at each time step for the standard foam is found by integration of the difference between the heat release rate terms inside the brackets in Eq. 19. The value of I for the foam, in the composite to be predicted, is determined from the running integral of the calculated heat release rate contribution of that foam. After the complete heat release rate curve for the foam is predicted using this procedure, the heat release rate of the fabric is added to yield the heat release rate of the composite as indicated in Eq. 20.
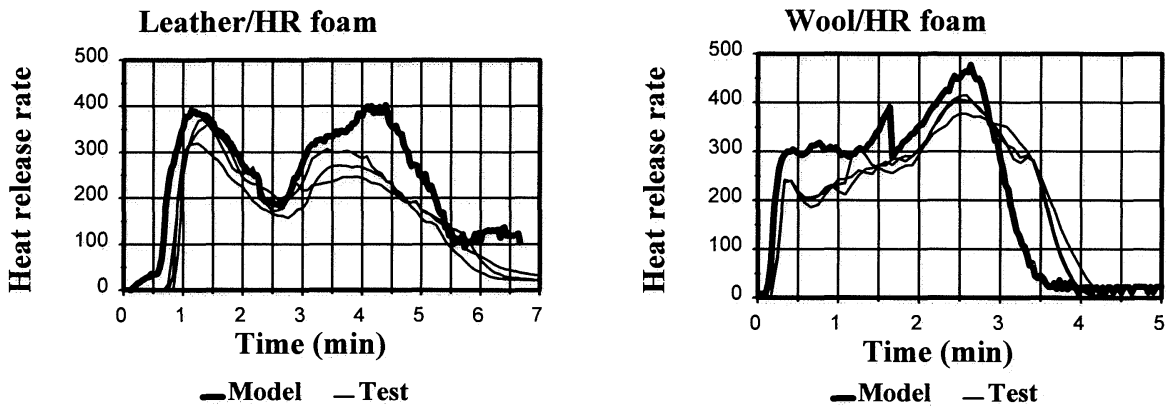

FR back-coated acrylic/HR foam
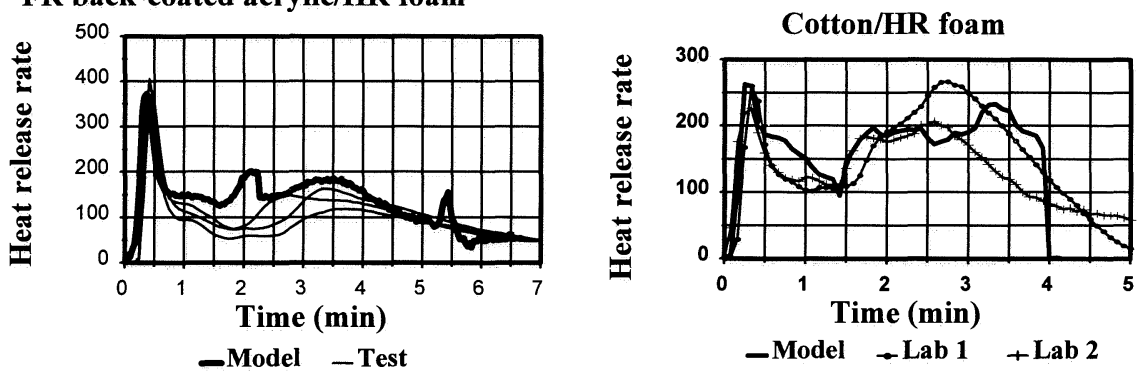

FIGURE 4. Comparison of predicted and measured heat release rates $\left(\mathrm{kW} / \mathrm{m}^{2}\right)$ of various fabrics over high resilience polyurethane foam.

\section{COMPARISON}

The predicted and measured heat release rate curves for four furniture composites consisting of different fabrics over HR foam are shown in Figure 4. The data on the components came from 
laboratories in Denmark and the UK. The comparison data on the composites was generated in a German laboratory. For comparison between the testing laboratories, the heat release rate of the cotton/HR foam composite measured in France is also shown. This data was all obtained on the CBUF program [1]. The model is in good qualitative agreement with the data although there are significant quantitative differences in some parts of the curves. In addition to the assumptions and approximations made in the model there are repeatability and reproducibilty considerations regarding the test data taken on both the components and the composites. Notice the variation of the test data for the three replicates at 2.5 minutes for the composite with the FR back coated acrylic fabric.

In the case of the cotton/HRR composite the agreement with the model and the test data was as good as the agreement between two different laboratories using the standard Cone calorimeter testing protocol. While there are some distinctive shape differences, the first peaks and the minimum between the peaks of all three curves are similar. The second peak of the calculated curve is in between the two peaks of the measured curves. The same is true of the 300 second average which is needed for the full scale correlation formula. This is based on an exposure of $35 \mathrm{~kW} / \mathrm{m}^{2}$ and is equal to 140,148 and $135 \mathrm{~kW} / \mathrm{m}^{2}$ for the calculation, Lab 1 and Lab 2 respectively. The abrupt drop at the end of the calculated curve is due to an automatic correction built into the computer program to insure that the total heat released by the foam in the composite being predicted is equal to its value in the bare foam component test. This correction only impacts the very end of the calculated heat release rate curves.

\section{DISCUSSION}

The procedure described in this paper only predicts the heat release rate of furniture composites in the Cone calorimeter. It does not, by itself, predict the full scale fire behavior of upholstered furniture. To execute the model it is necessary to have input data obtained in the Cone calorimeter on the heat release rate of (1) the fabric cover burning over a standard foam, (2) the fabric cover burning by itself with flow of methane passing through it to simulate the volatiles from a foam, and (3) the foam burning by itself with a methane flame above it. The testing protocols for obtaining these data are described in the CBUF final report [2]. The model is based on the properties of melting foams and does not apply to cotton batting and other char forming paddings. So far it has only been demonstrated for different types of polyurethane foams. It works for both melting and char forming fabrics. It is not necessary to know in advance whether the the fabric will melt or char.

The composite model could reduce the amount of testing required, because of all the composites that can be formed from a few components. The burden of the testing could also be lifted from the furniture manufacturers. It could be done by the material suppliers. The necessity of testing a particular fabric or foam over again as a part of a different composite by every new user could be avoided. The material manufacturer could develop formulas for the effects of various material parameters on the heat release rate of their products. Then much of the testing could be replaced by simple calculations. Using the composite model along with a furniture fire model, a furniture item could be designed with its fire performance taken into account. 


\section{CONCLUSIONS}

A procedure was developed for predicting the approximate shape and magnitude of the heat release rate curves in the Cone calorimeter for furniture composites using input data from modified tests on the furniture components. The predictions agreed reasonably well with the data obtained on the composites. However, more work is needed on a broader range of materials.

The composite model is based on the following three major findings during this research.

(1) By passing methane through a fabric burning by itself, it can be made to burn approximately like it would if it were part of a composite.

(2) If bare polyurethane foam is exposed to a range of external fluxes, the resulting heat release rate curves can be collapsed into one generalized curve by dividing each curve by its peak heat release rate and plotting it against its integrated heat release up to that time.

(3) The heat flux that is passed on by a burning fabric to an underlying foam can be assessed by measuring the heat release rate contribution of a standard foam of known sensitivity that is covered by that fabric. The contribution of the standard foam is equal to the heat release rate of this composite minus the heat release rate of the fabric burning alone.

\section{ACKNOWLEDGEMENTS}

The author wishes to thank Björn Sundström at SP for his support and critique; Ingrid Wetterlund and Lars Pettersson at SP for their help in the experiments leading to the development of the model and the component test procedures; Jimmie Beckerlee at DBI and Sheila Monro at FMC for the Cone calorimeter tests on the components; and Stefan Lehner at FMPA and Alain Sainrat at LNE for the Cone calorimeter tests on the composites.

\section{REFERENCES}

1. ASTM E 1354 - 94, "Standard Test Method for Heat and Visible Smoke Release Rates for Materials and Products Using an Oxygen Consumption Calorimeter," American Society for Testing and Materials, 1995

2. Fire Safety of Upholstered Furniture - the final report on the CBUF research programme, ed. B. Sundström, Interscience Communication, Limited, London, 1995.

3. ASTM E 1474 - 95, "Standard Test Method for Determining the Heat Release Rate of Upholstered Furniture and Mattress Components and Composites Using a Bench Scale Oxygen Consumption Calorimeter," American Society for Testing and Materials, 1995

4. Babrauskas, V. and Wetterlund, I, "Fire Testing of Furniture in the Cone Calorimeter-The CBUF Test Protocol," SP Report 1994:34. Available from the Swedish National Testing and Research Institute, Fire Technology, PO Box 857, S-501, 15 Borås, Sweden 